\title{
Digital holography-coherent optics of the 21st century: introduction
}

\author{
Ting-Chung Poon, Toyohiko Yatagai, and Werner Jüptner
}

The electron is dead, long live the photon. This opinion was pointed out at the beginning of this century to underscore the new significance of optics. However, one has to recognize that the rebirth of optics is due to the extraordinary development of electronics in the past 30 years. In particular, electronic data processing has been developing in a fascinating, enabling way during this time. Digital holography or electronic holography is an excellent example of this. In 1986 one of us (Jüptner) gave a talk on robot vision and mentioned the possibility of three-dimensional (3D) vision for robots by means of electronically processed holograms. Our colleagues were excited about this. However, at the time we had to acknowledge the absence of not only the optoelectronics necessary to capture the hologram but also of the high-power data processing needed to achieve nearly real-time processing of the holograms. Today we are close to realizing these tools. Digital holography is at the beginning of an era of use as a common tool in science and technology.

T.-C. Poon (tcpoon@vt.edu) is with the Department of Electrical and Computer Engineering, Virginia Tech, Blacksburg, Virginia 24061. T. Yatagai is with the Institute of Applied Physics, University of Tsukuba, Tsukuba, Japan. W. Jüptner is with the Bremen Institute für Angewandte Strahltechnik, Bremen, Germany.

0003-6935/06/050821-01\$15.00/0

(C) 2006 Optical Society of America
Digital holography introduces something very new to optical science: For the first time it is possible to measure the phase of a light wave. All the pioneers in optics in the past compared only two coherent waves and their phases. So their results were in the form of phase differences. Digital holography allows us to determine the phase of a light field as well the intensity; i.e., the whole wave field can be measured and stored in a computer. This means that any experiment can be simulated and, what's more, we can simulate experiments that cannot be performed in reality (e.g., add a negative intensity). This capability expands the horizons of holography to include its use as a scientific and technological tool. The measurements of shape, deformation, and particle distributions, as well as microscopic measurement according to the dreams of Gabor, are becoming standard.

In this feature issue we compile a collection of key scientific results that demonstrate the new developments on and with digital holography. The issue covers holographic microscopy and optical trapping and tweezing and includes applications such as new methods of fast and precise evaluation, measurement of fast-moving objects, and particle sizing to beam shaping. We are convinced that these examples will give rise to new ideas for the development of this interesting field of coherent optics.

We thank Joseph Mait for suggesting this feature issue and Keith Jackson for his help in ensuring the timely production of this special issue. 